\title{
Concept and status of Concentrating Solar Power systems
}

\author{
R. Pitz-PAAL $\left(^{*}\right)$ \\ DLR Institute of Solar Research - Linder Höhe, 51147 Cologne, Germany
}

\begin{abstract}
Summary. - Development of Concentrating Solar Power (CSP) systems has started about 40 years ago. A first commercial implementation was performed between 1985 and 1991 in California. However, a drop in gas prices caused a longer period without further deployment. It was overcome in 2007 when new incentive schemes for renewables in Spain and the US enabled a commercial restart. In 2017, almost 100 commercial CSP plants with more than $5 \mathrm{GW}$ are installed worldwide. This paper describes the physical background of CSP technology, its technical characteristics and concepts. Furthermore, it discusses system performances, cost structures and the expected advancement.
\end{abstract}

\section{1. - Introduction}

Concentrating Solar Thermal Power (CSP) systems use high-temperature heat from concentrating solar collectors to generate power in a conventional power cycle instead of — or in addition to — burning fuel. The thermal energy accumulated by the concentrating collectors can be stored at low cost in a thermal energy storage (e.g. a tank

$\left(^{*}\right)$ E-mail: robert.pitz-paal@dlr.de 


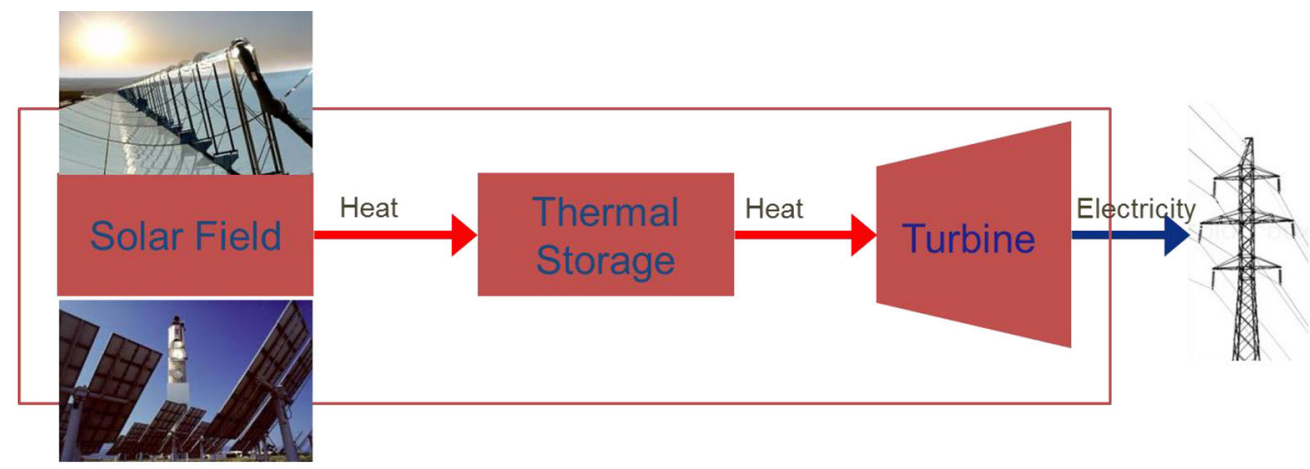

Fig. 1. - The concept of Concentrating Solar Power Systems (CSP) [source DLR].

with molten salt) before it is provided to a conventional power cycle (fig. 1). Thus, electricity production can be shifted to periods of high load demand and the technology is capable to complement the production from wind and photovoltaic as depicted in fig. 2 . Firm supply can be guaranteed if additional fuel (e.g. from biomass) is used during the uncommon periods when the thermal storage is empty.

\section{2. - Technical background of CSP}

$2 \cdot 1$. Solar resource. - Only direct radiation can be concentrated in optical systems (fig. 3). In order to achieve significant concentration factors, Sun-tracking is required, adding complexity to the system. Therefore, CSP is most suitable for centralized power production, where maintenance can be performed efficiently, and in areas with high, non-volatile direct solar radiation levels. The concentration of sunlight is achieved by mirrors directing the sunlight onto a heat exchanger (receiver/absorber), transferring the absorbed energy to a heat transfer fluid.

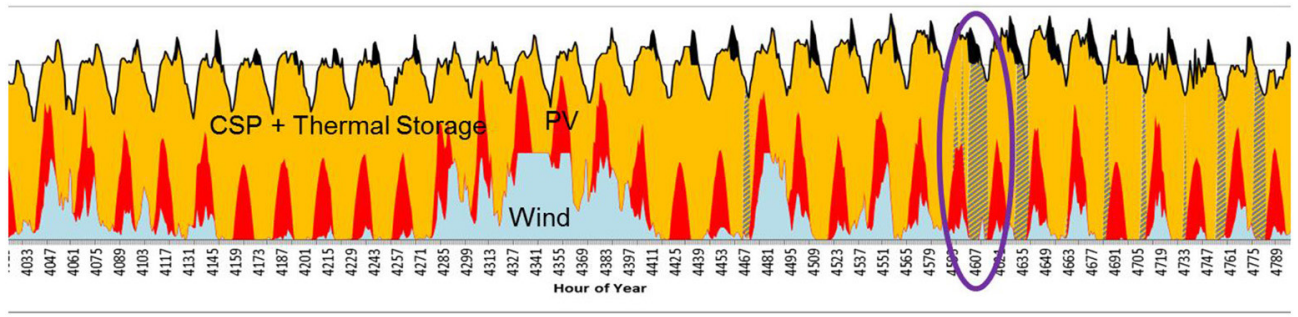

Fig. 2. - Electric load matched by a mix of wind, PV and CSP with thermal storage: During periods indicated in gray co-combustion in CSP systems is required to match the load [source DLR]. 


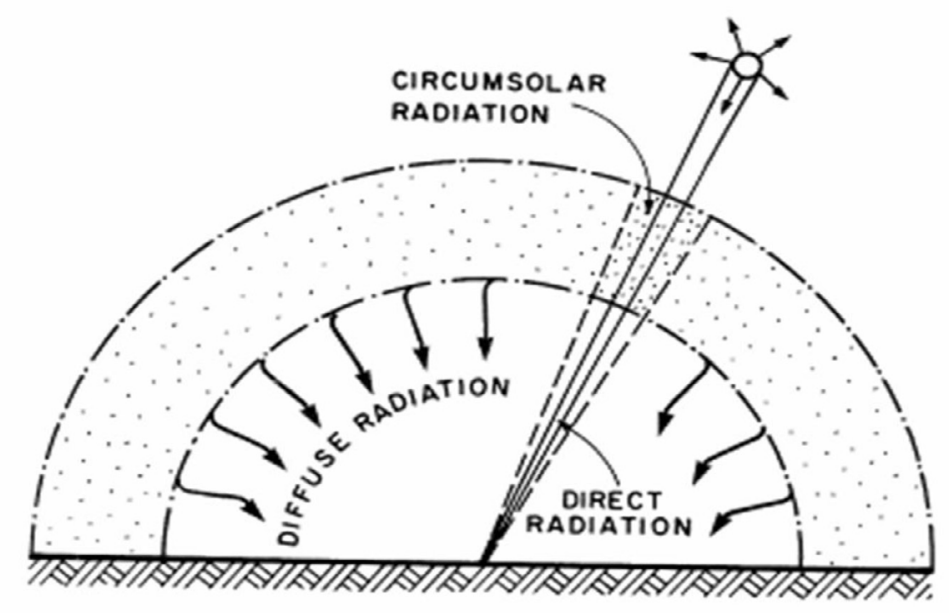

Fig. 3. - Definition of direct, diffuse and circumsolar radiation. Only direct irradicance can be used for CSP [1].

$2 \cdot 2$. Concentration of sunlight. - Radiation power (unit: Watt) that is emitted from a point-like source is diluted with increasing distance from its source. This means that the power density $E$ (unit: $\mathrm{W} / \mathrm{m}^{2}$ ) is reduced with increasing distance, because the emitted power is distributed over a larger surface. Concentration of radiations aims to increase the power density $E$ of the radiant energy for optimized utilization.

A generic concentrator (fig. 4) consists of a concentrator entrance aperture area $A$ (unit: $\mathrm{m}^{2}$ ) in which the radiant energy enters and an exit aperture $A^{\prime}$ from which the radiation energy leaves. Assuming no losses in the concentrator, the law of conservation of energy leads to the concentration factor $C$. $C$ is the ratio of the outgoing to the incoming energy density and can be described as the inverse ratio of the respective aperture areas:

$$
C=E^{\prime} / E=A / A^{\prime}
$$

As can be derived from complex thermodynamic considerations, ideal concentrators conserve a quantity called entendue. In this case, entendue is the product of aperture area and sinus squared of the radiation cone's half angle $\theta$ :

$$
A \sin ^{2} \theta=A^{\prime} \sin ^{2} \theta^{\prime}
$$

From this law, the concentration ratio of an ideal concentrator results as

$$
C=A / A^{\prime}=\sin ^{2} \theta^{\prime} / \sin ^{2} \theta
$$

For a given opening angle $\theta$, the maximum concentration is achieved if $\sin ^{2} \theta^{\prime}=1\left(\theta^{\prime}=\right.$ $\left.90^{\circ}\right)$. 


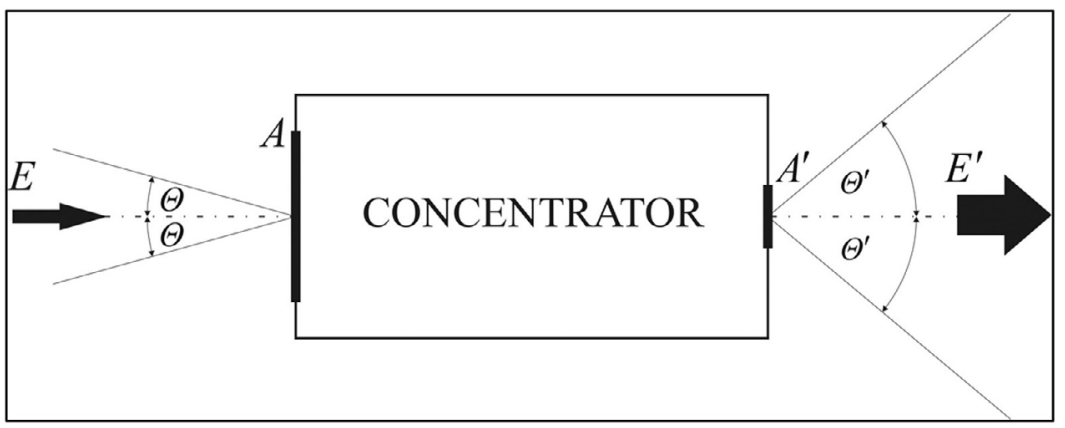

Fig. 4. - Schematic of a solar concentrator [1].

Thus, for the Sun's half angle of $4.653 \mathrm{mrad}$ a maximum concentration ratio of $1 / \mathrm{sin}^{2}$ $(4.653 \mathrm{mrad}) \approx 46200$ can be achieved.

In practice, imaging mirror concentrators rather than lens concentrators are applied as primary concentrators for solar high-temperature applications. Imaging mirror concentrators have better outdoor durability and lower specific costs. Their design generally approximates the parabola shape in a continuous or segmented way (fig. 5). The image of the Sun as generated by such a mirror reflector is blurred by the optical imperfections of the parabola concentrator concept itself (aberration) and by imperfect surface characteristics. The resulting image consists of superimposed images of individual concentrator segments having elliptical shapes with diameters depending on the opening angle of the Sun's disk $\alpha_{D}$, the distance between reflector and focal point $r_{r}$, and on the opening angle of the parabola $\Psi$.

The concentration factor of the parabola is determined by the largest of all superimposed images $A_{i m}$ that comes from the rim of the parabola:

$$
C=\frac{A_{a p}}{A_{i m}}
$$

with

$$
A_{a p}=\pi r_{r}^{2} \sin ^{2} \psi \quad \text { and } \quad A_{i m}=\frac{\pi}{4} \frac{r_{r}^{2} \alpha_{D}^{2}}{\cos ^{2} \psi}
$$

leads to

$$
C=\frac{4}{\alpha_{D}^{2}} \sin ^{2} \psi \cos ^{2} \psi=46200 \sin ^{2} \psi \cos ^{2} \psi
$$

The maximum concentration achievable by an ideal parabola does not reach the theoretical limit of 46200 , but only $1 / 4$ of it at an opening angle of the parabola $\Psi$ of $45^{\circ}$ :

$$
C_{\max }=46200 \cdot 0.5 \cdot 0.5=11550 .
$$




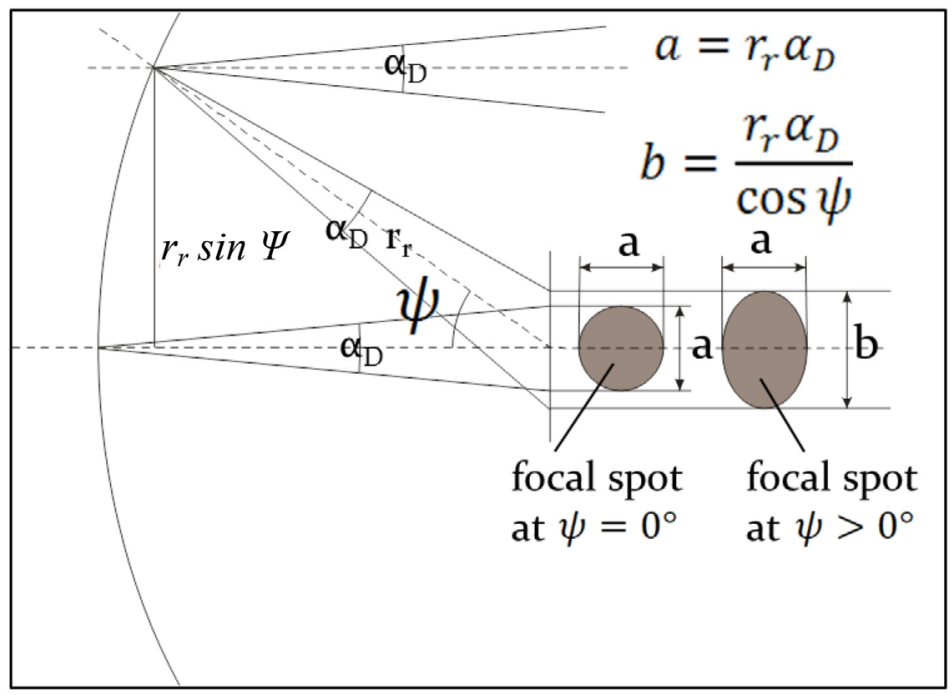

Fig. 5. - Size of the focal spot of an ideal parabola [source DLR].

The superposition of the images can often be well approximated by a Gaussian distribution. Thus, the energy density at the exit aperture of a parabola concentrator is not constant, but varies from a peak value to zero at an infinite distance. For practical application it is reasonable to use only the central part of the Gaussian profile and discard the rest. The amount of discarded energy is often an outcome of an economic optimization process with typical values ranging from $5 \%$ to $10 \%$.

2:3. Conversion of concentrated sunlight to heat. - The device used in hightemperature solar concentrators for the conversion of concentrated solar radiation to heat is called receiver. It is designed to absorb the concentrated solar radiation and to transfer as much energy as possible to a heat transfer fluid. Losses have several origins: The absorbing surface may not be completely black, the surface emits thermal radiation to the environment (due to its elevated temperature) and convection as well as conduction occur. The thermal efficiency of the receiver $\eta_{\text {th }}$ is defined by the ratio of the useable heat per second to the incoming solar radiation in the aperture. As shown in fig. $6, \eta_{\text {th }}$ is decreasing with increasing temperatures due to higher heat losses. On the other hand, higher concentration factors lead to higher efficiencies and both convection and conduction losses are of minor importance at high concentration factors.

$\mathbf{2}$ 4. Thermodynamic cycles. - In high-temperature solar concentrator systems, the absorbed energy is often converted into mechanical energy and subsequently to electricity in a power cycle. A part of that cycle is an engine that continuously transfers heat to mechanical energy. Steam or gas turbine cycles are common examples used in fossil power plants to generate electricity from the heat input provided by the combustion of 


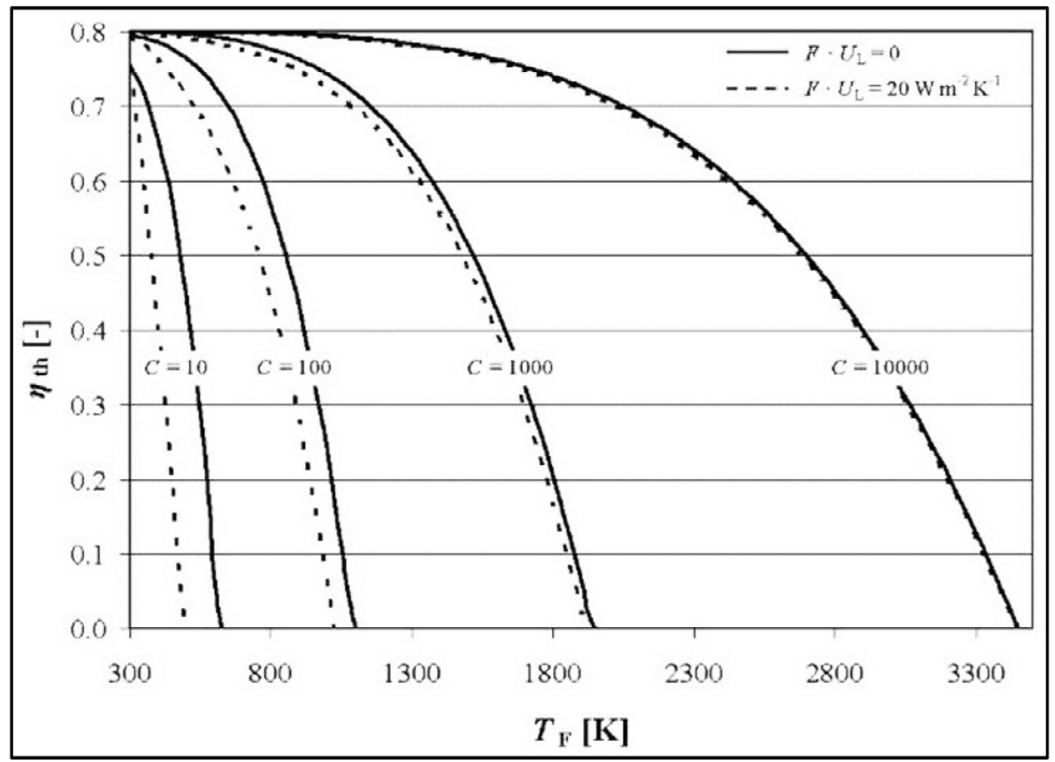

Fig. 6. - Efficiency of a solar receiver as a function of the fluid temperature, the concentration ratio $C$ and the effective convective heat loss coefficient $F U_{L}[1]$.

fuels (fig. 7a). However, thermodynamic principles forbid that all heat input is completely transferred to electricity, because a fraction of it must be removed at a lower temperature level.

a)

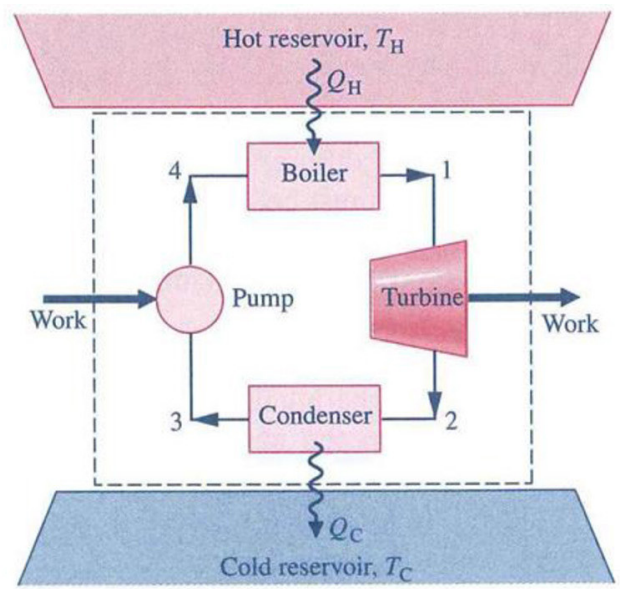

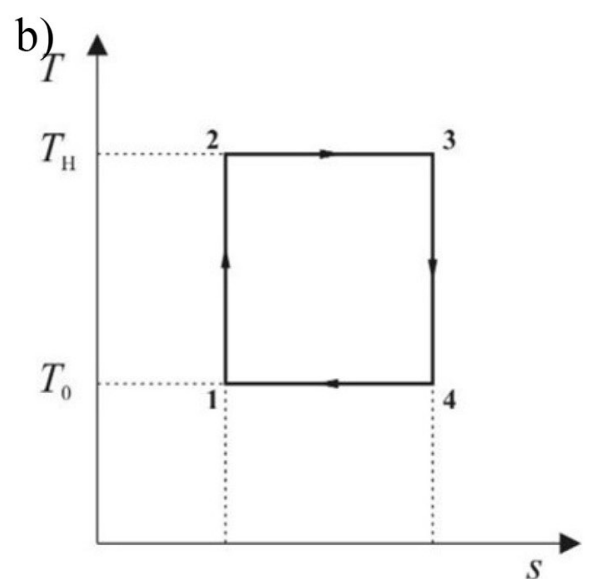

Fig. 7. - Schematic of an ideal Carnot Cycle (a) and state changes in the $T$-S representation (b) [source DLR]. 
In each generic power cycle, a working fluid undergoes a number of changes in state. In order to force the system from one state to the next, heat or work must be delivered to or extracted from the fluid. Depending on the kind of working fluid and the state changes, many different power cycles can be set up. An example is the Carnot cycle, an idealized reversible process that consists of two isothermal $(2 \rightarrow 3$ and $4 \rightarrow 1)$ and two isentropic $(1 \rightarrow 2$ and $3 \rightarrow 4$ ) changes in state. In fig. $7 \mathrm{~b}$, the corresponding $T / S$-representation is shown, where $S$ is the specific entropy of the fluid.

The fluid receives heat at the upper temperature $T_{\mathrm{H}}$ and at the lower temperature $T_{0}$ heat is released. The cycle efficiency $\eta_{\mathrm{C}}$ describing the fraction of heat input $Q_{\text {in }}$ that is converted to work $W$ is independent of the working fluid and can be calculated to be

$$
\eta_{\mathrm{C}}=\frac{W}{Q_{\text {in }}}=1-\frac{T_{0}}{T_{\mathrm{H}}}
$$

Today, there are no power cycles specifically developed for high-temperature solar concentrating systems but conventional fossil fuel driven power generation systems are adapted to solar applications. The most relevant ones are steam turbine cycles, gas turbine cycles and Stirling engines. Currently, steam cycles are the most common choice in commercial CSP projects. They are suited to power levels beyond $10 \mathrm{MW}$ and temperatures of up to $600{ }^{\circ} \mathrm{C}$. Steam cycles can be coupled to parabolic trough, linear Fresnel and central receiver systems. Stirling engines are used for small power levels (up to some $10 \mathrm{~kW}_{\mathrm{el}}$ ). Gas turbines offer the potential to exploit higher temperatures than steam cycles (up to $1200^{\circ} \mathrm{C}$ ) and cover a wide range of capacities from some hundred $\mathrm{kW}$ el to some $10 \mathrm{MW}_{\mathrm{el}}$. At high power levels, gas turbine cycles may be combined with steam cycles to highly efficient combined cycle systems promising to produce the same power output with a $25 \%$ smaller solar collector field. Up to now, solar gas turbines have been used in experimental facilities only.

25. Thermal energy storage. - Like domestic hot water systems, CSP systems have the important advantage of optional thermal energy storage systems (e.g. tanks with molten salt), allowing the operation of the plant to continue during transient clouds or after sunset. Thereby, a predictable power supply to the electricity grid can be achieved. In contrast to other renewable systems with electric storage, for which the inclusion of storage capacity always leads to far higher investments and higher electricity prices, CSP systems with storage are potentially cheaper than CSP systems without storage. This becomes clear when comparing a solar power plant without storage of, e.g., $100 \mathrm{MW}_{\mathrm{el}}$ capacity, operated approx. 2000 equivalent full-load hours per year at a typical site, to a system with half the capacity $\left(50 \mathrm{MW}_{\mathrm{el}}\right)$ but the same size solar field and a suitable thermal energy storage (fig. 8). In this case, the smaller power block is used for 4000 equivalent full load hours so that both systems can produce the same amount of electricity per year. Assuming low storage costs, the investment in the second system could potentially be lower than in the first. 


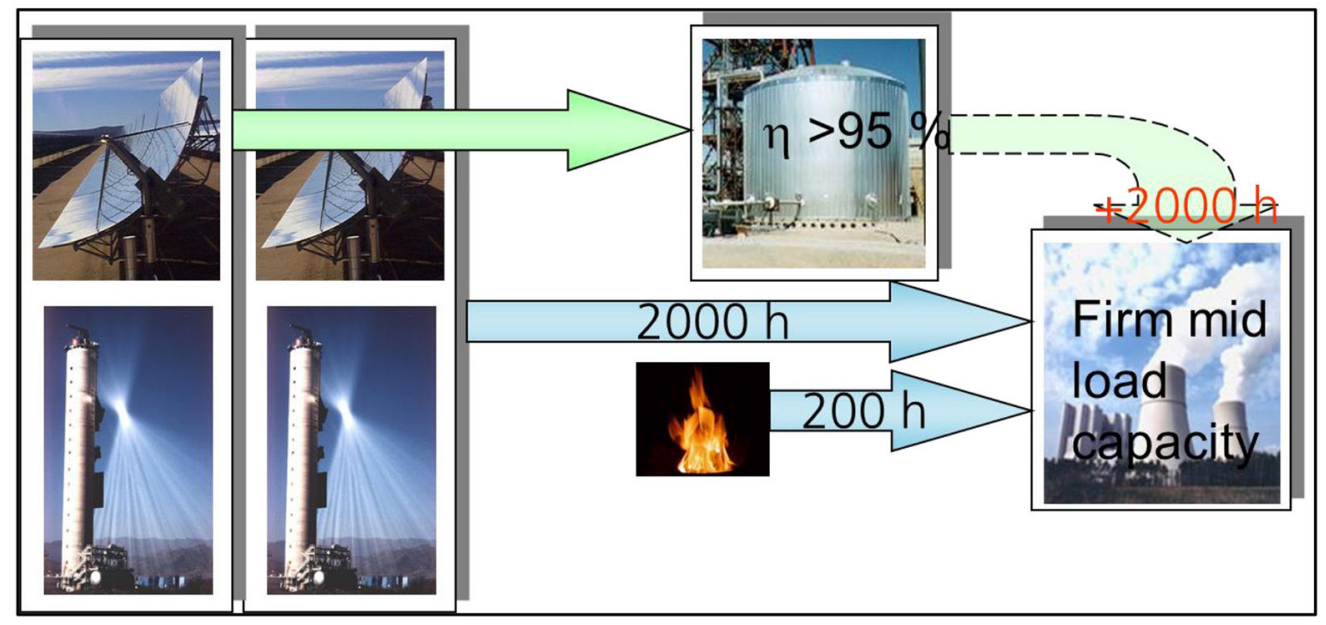

Fig. 8. - Integration of a thermal storage in a CSP system may lead to lower specific electricity cost (see text) [source DLR].

2*6. Optimizing CSP-systems. - According to the principles of thermodynamics, power cycles convert heat to mechanical energy more efficiently at higher temperatures. However, the collector efficiency drops at higher absorber temperatures due to higher heat losses. Consequently, for any given concentration factor there is an optimum operation temperature at which the highest conversion efficiency from solar energy to work is achieved. With rising concentration higher optimum efficiencies are achievable. Figure 9 illustrates this characteristic assuming an ideal solar concentrator combined with an ideal Carnot power cycle. If the spectral absorption characteristics of the absorber are perfectly tailored to maximise absorption in the solar spectrum, but avoid thermal radiation losses in the infrared part of the spectrum (selective absorber), additional efficiency gains can be expected in particular at lower concentration factors.

In practice, the optimum operation temperatures will be lower than these theoretical figures, because power cycles with Carnot performance and ideal absorbers do not exist. Furthermore, the impact of frequent operation under part-load conditions throughout the year on the efficiency of the system has to be considered.

\section{3. - Technical concepts}

CSP systems can be distinguished by the arrangement of their concentrator mirrors: Line focusing systems like parabolic troughs or linear Fresnel systems (fig. 10, left and middle) only require single axis tracking in order to concentrate the solar radiation onto an absorber tube. Concentration factors of up to 100 can be achieved in practice. Point focusing systems like central receiver systems (fig. 10 right) can achieve concentration factors of several 1000 at the expense of two-axis tracking. They use a large number of 


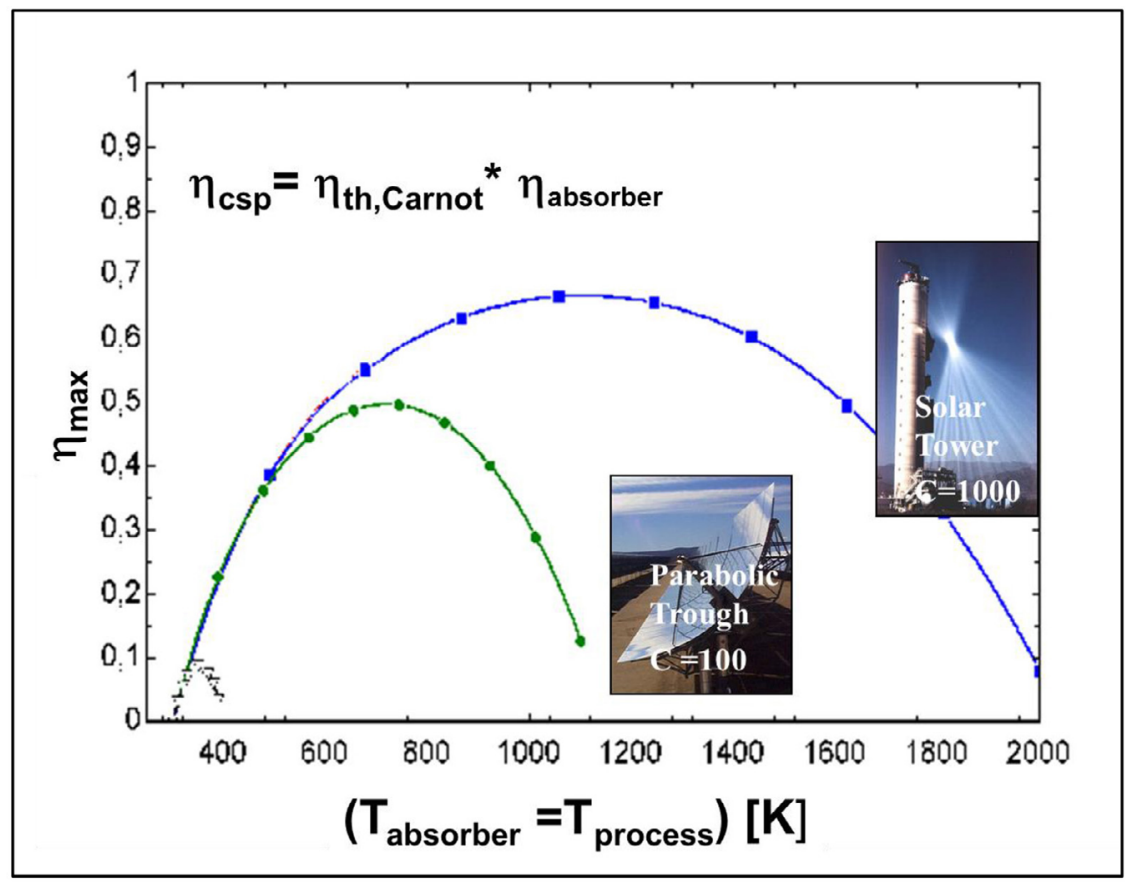

Fig. 9. - System efficiency as a function of upper fluid temperature for different solar concentrating systems [source DLR].

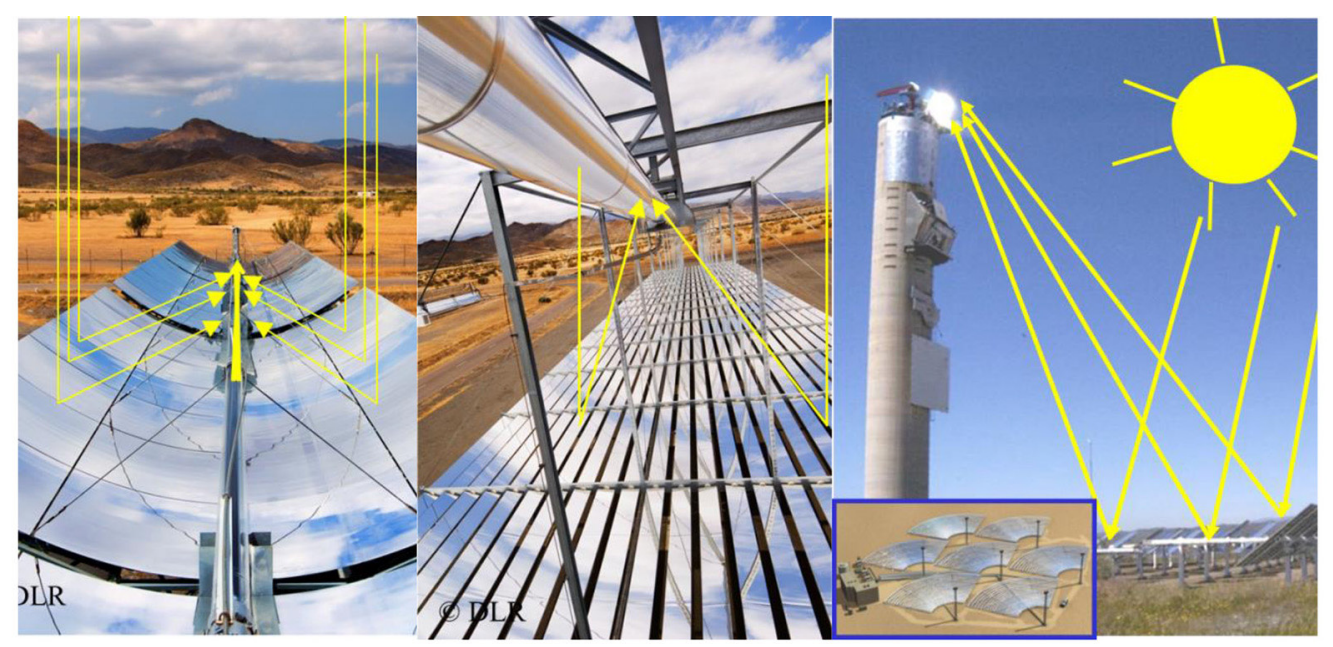

Fig. 10. - Schematic of a parabolic trough collector (left), linear Fresnel (center) and solar tower system (right) [source DLR]. 


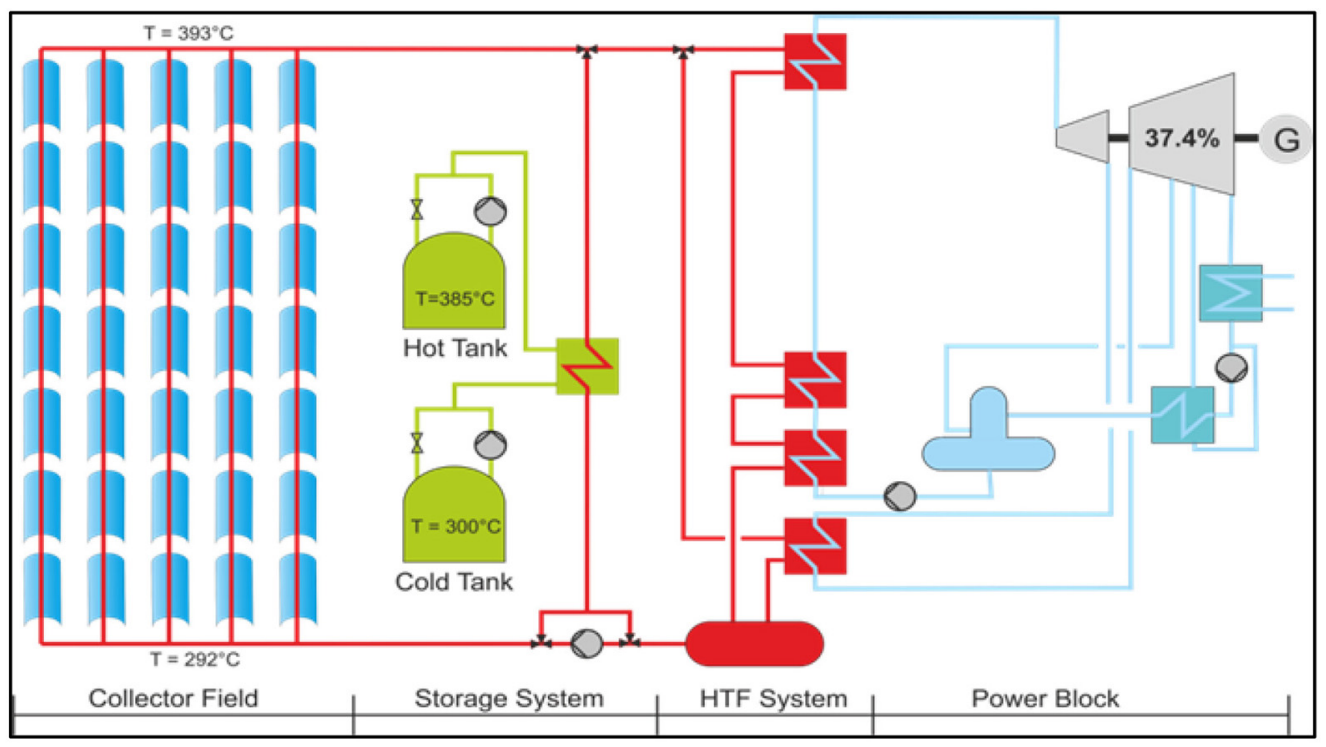

Fig. 11. - Schematic of a system based on parabolic trough collector using thermal oil as heat transfer fluid and 2 tank molten salt storage system [2].

individually tracking heliostats to concentrate the solar radiation onto a receiver located on the top of a central tower.

3`1. Line focusing systems. - Most of today's commercial CSP power plants $(\Sigma>$ $3 \mathrm{GW}_{\mathrm{e}}$ ) are based on the design of the ANDASOL parabolic trough power plant (fig. 11) put in operation in Spain in November 2008. The plant design integrates three different fluid cycles: a eutectic mixture of $73.5 \%$ diphenyl oxide and $26.5 \%$ biphenyl is used as heat transfer fluid in the collector field. It is operated between $290^{\circ} \mathrm{C}$ and $390^{\circ} \mathrm{C}$ (the upper temperature limit of this thermal oil) and provides the collected energy through heat exchangers either to the thermal storage cycle or to the power cycle. The power cycle uses water steam like in conventional power plants whereas a eutectic mixture of $\mathrm{KNO}_{3}$ and $\mathrm{NaNO}_{3}$ acts as a storage medium at almost the same temperature range as the collector fluid. The mixture is chosen as it is about 4 times cheaper than thermal oil. The salt is contained in two tanks, one on a lower temperature of about $290^{\circ} \mathrm{C}$ and, after being heated up in the heat exchanger, in a hot tank at almost $390^{\circ} \mathrm{C}$. The salt mixture material is available extensively in nature and used also as fertilizer with a capacity of many 100 of thousands tons per year. As it freezes at $238^{\circ} \mathrm{C}$, it needs to be kept significantly above that temperature during the lifetime of the power plant. The storage density of such a system is approximately $78 \mathrm{kWh}_{\mathrm{th}} / \mathrm{m}^{3}$ or $42 \mathrm{kWh}$ th $/ \mathrm{t}$. To provide a $100 \mathrm{MW}_{\mathrm{e}}$ trough power plant with a 6 hour of storage capacity about $38000 \mathrm{t}$ of salt are required. The largest commercial installation that uses such a storage system was put in operation in October 2013. It is the $280 \mathrm{MW}_{\mathrm{e}}$ Solana plant in Arizona (US) equipped 
with a 6 hours storage equivalent to $4400 \mathrm{MWh}_{\text {th }}$ of storage capacity distributed in 6 pairs of tanks of hot and cold salt each about $38 \mathrm{~m}$ in diameter and $14 \mathrm{~m}$ high. The heat losses of such tanks are significantly below $3 \%$ of the energy throughput and even two weeks of interruption does not require active heating for freezing protection of the storage tanks. Heat exchangers, pumps, valves, and pipes need to be equipped with electrical trace heating to avoid freezing problems during start-up. Such a power plant concept can achieve annual net solar-to-electric efficiency figures of up to $15 \%$.

This concept has a number of limitations in terms of power plant efficiency and storage density that led to the development of alternative designs. First, the synthetic oil heat transfer is rather expensive and limited to approximately $400{ }^{\circ} \mathrm{C}$. This confines the potential life steam conditions in the power block and thus the efficiency of the power cycle to values below those of fossil fuel driven steam power plants. Moreover, it limits the amount of heat that can be stored in the salt as it depends on the temperature difference between hot and cold tank. Steam, also used in the power cycle, has been investigated as an alternative heat transfer fluid. However, the integration into a storage system is difficult as the phase change characteristics of the steam during evaporation leads to a temperature profile that does not correspond to the sensible heating of the salt mixture. Using salt not only as a storage medium, but also as heat transfer fluid may overcome many of the limitations as the operation temperature of the salt can be extended to $550{ }^{\circ} \mathrm{C}$ which is a more typical operation temperature of conventional steam cycle power plants. Such a replacement would also avoid the expensive thermal oil cycle. The challenge, however, is to avoid freezing of the salt in the extended collector field under any circumstances.

If such a concept is applied to a parabolic trough system (fig. 12) the molten salt needs to be circulated through the whole collector field ( $>100 \mathrm{~km}$ of piping) and a complete draining of the systems (e.g. overnight) does not seem to be an attractive option for freezing protection. In order to prevent the salt from freezing in the absorber tubes, a continuous recirculation, using salt from the cold tank and redirecting it back to the cold tank, poses a solution. Any drop in temperature of the cold tank will be compensated by small amounts of solar-heated molten salt from the hot tank. Once the hot tank is empty the heat will be provided by the auxiliary heater. Typically the auxiliary heater is not used after a sunny day. As molten salt always circulates through the solar field, it requires energy for pumping. The additionally installed electric heating devices in the solar field are only installed for emergencies and will not be used in a regular operation of the plant.

3·2. Solar tower systems. - In solar tower systems, a field of individually tracked mirrors (heliostats) concentrates the solar radiation on a heat exchanger located on top of a central tower (fig. 10 right). In such a point-focusing system, higher concentration factors $(\approx 1000)$ can be archived compared to parabolic trough technology $(\approx 100)$ that generates a line-focus. The collection of the energy is not performed by an extended piping circuit of more than $100 \mathrm{~km}$ length (including the parabolic trough receiver tubes) like in a parabolic trough field but rather by transferring the radiation energy as light 


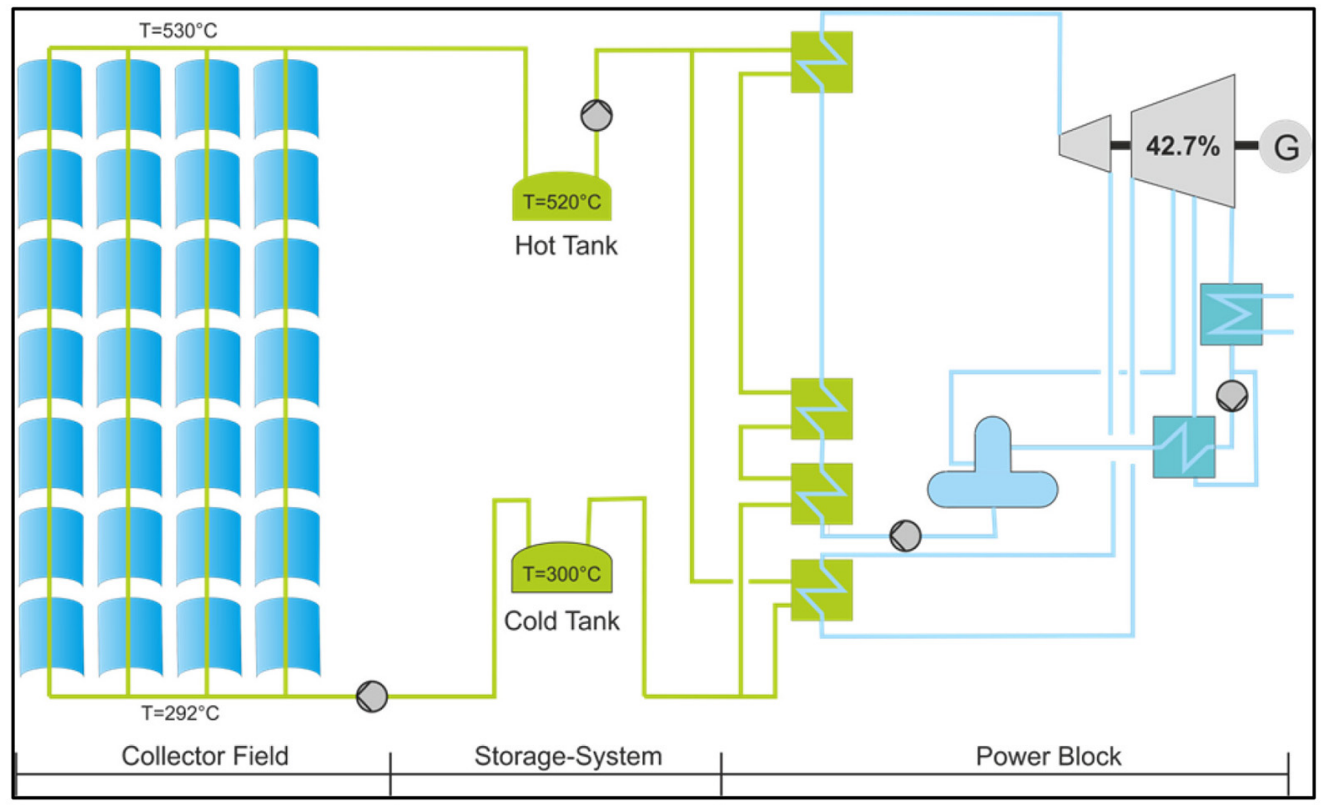

Fig. 12. - Schematic of a system based on parabolic trough collector using molten salt as heat transfer fluid and a 2 tank molten salt storage system [2].

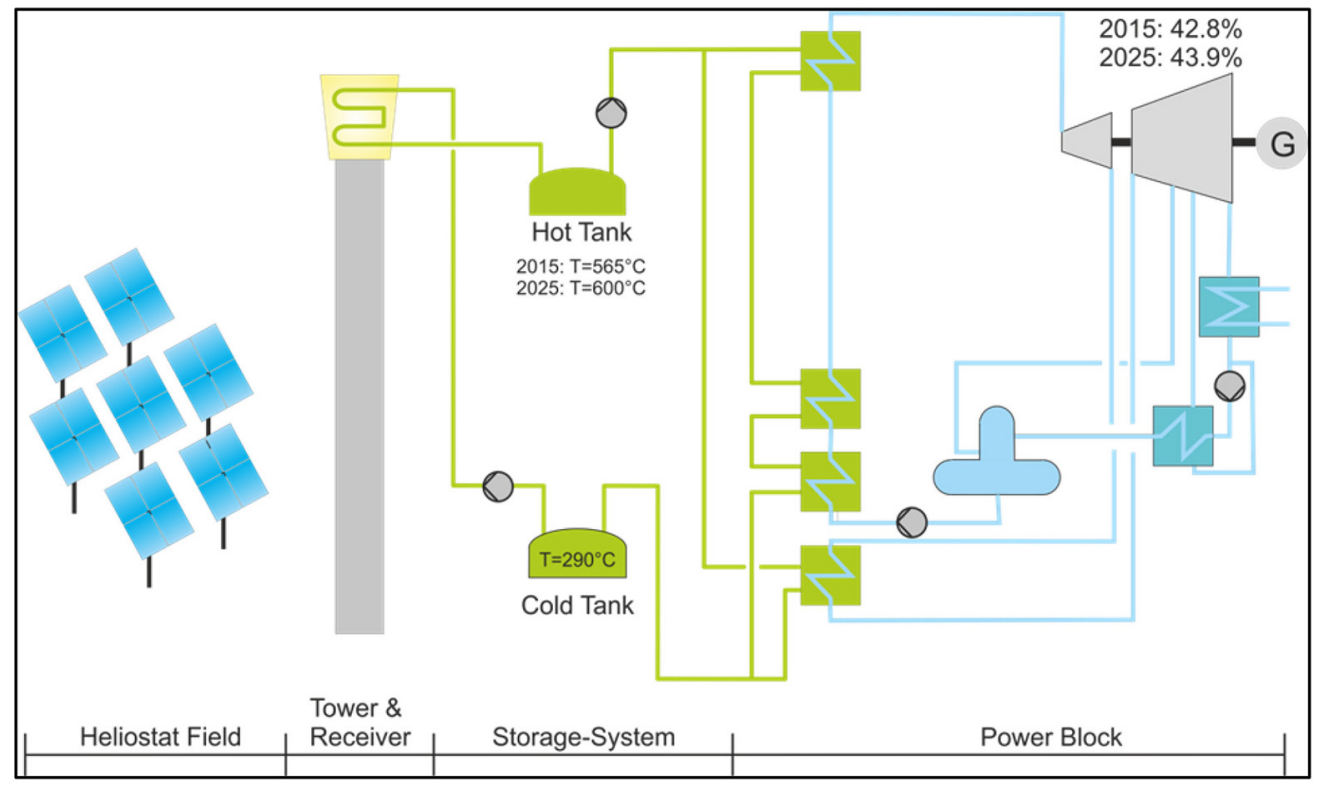

Fig. 13. - Schematic of a solar tower system using molten salt as heat transfer fluid and a two-tank molten salt storage system [2]. 
TABLE I. - Data for reference system concepts to evaluate performances and costs for different site conditions [3].

\begin{tabular}{lccc}
\hline Design parameters in 2015 & Unit & Parabolic Trough & Solar Tower \\
\hline Site & & Ouarzazate, Morocco \\
Direct normal irradiation (DNI) & {$\left[\mathrm{kWh} /\left(\mathrm{m}^{2} \cdot \mathrm{a}\right)\right]$} & $2017 / 2558 / 2935$ \\
Solar collector / heliostat & & Ultimate Trough@ & Stellioß \\
Heat transfer fluid (HTF) & thermal oil & molten salt \\
Storage medium & & molten salt & molten salt \\
Maximum HTF temperature & {$\left[{ }^{\circ} \mathrm{C}\right]$} & 393 & 565 \\
Thermal energy storage capacity (full load & {$[\mathrm{h}]$} & 7.5 & $9^{\text {(a) }}$ \\
hours) & & & 150 \\
Gross electrical output & {$[\mathrm{MW}]$} & 160 & 150 \\
\hline
\end{tabular}

(a) Result of LCOE optimisation.

to a central location. This limits the piping length to a couple of $100 \mathrm{~m}$ necessary to transport the heat transfer fluid from the top of the tower to the storage and steam generator system located on the ground (fig. 13). This configuration has the benefit that gravity supports the draining of the complete salt fluid back to the storage tanks during times when operation is not scheduled (e.g. overnight). This reduces the auxiliary electric power requirements to heat the pipes in order to avoid freezing, in the case that molten salt is used as heat transfer and storage fluid. However, such a design also shows some significant drawbacks: A liquid salt column of $100 \mathrm{~m}$ equals 19 bar pressure. As the tower in a commercial solar system is typically more than $150 \mathrm{~m}$ tall and the pressure in the storage tanks at the ground level should be kept close to atmospheric pressure to limit construction costs, the salt circulation system cannot be designed as a closed circuit in which only friction losses need to be overcome by the pump. In an open cycle, the energy required to lift the salt to the tower top needs to be invested.

\section{4. - Performance}

In fig. 14, the annual energy flow in a CSP line focusing system is depicted qualitatively. Energy flows in tower systems differ only slightly. Optical losses associated with the solar field are caused by geometrical imperfections, surface impairments (e.g. dust) and extinction (aerosols). Thermal losses are linked to heat losses of high-temperature energy flows and to auxiliary warming-up effects. Power block losses are due to thermodynamic constraints that require heat removal of the cycle at low temperatures.

Performance and cost figures of CSP systems depend strongly on the power plant designs with respect to its size, its capacity factor (storage) as well as plant location 


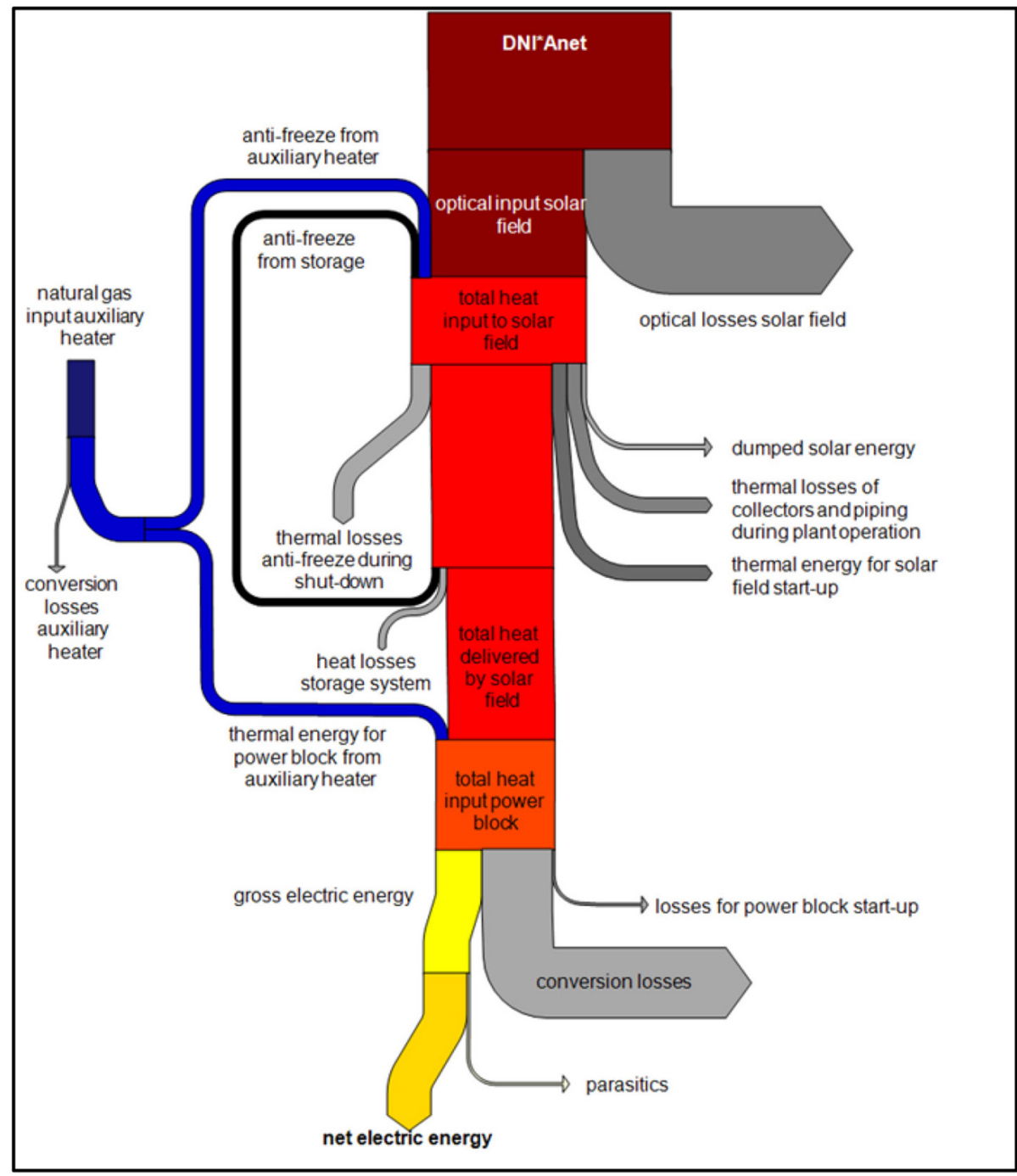

Fig. 14. - Sankey diagram for energy flows in a molten salt line focusing system [3].

and solar resource. In order to discuss this effect, the boundary conditions of different solar reference plant concepts investigated by IRENA [3] are summarized in table I. The concepts comprise of trough and tower technology with a certain capacity and storage size investigated for three different solar conditions that are representative for typical CSP markets.

Performance calculations of those concepts have been conducted with the numerical tool Greenius (B) [3] and are shown for trough systems in table II and for tower systems in table III. Advancements in technology until 2025 are considered (e.g. replacing thermal 
TABLE II. - Electric production and system performance figures of a $160 \mathrm{MW}_{\mathrm{e}}$ parabolic trough system for state of the art technology (thermal oil heat transfer fluid) and estimations for 2025 (molten salt heat transfer fluid) [3].

\begin{tabular}{c|ccc|cc|cr|}
\hline & \multicolumn{2}{|c|}{$\begin{array}{c}\text { Net electrical output } \\
{[\text { GWh / a }]}\end{array}$} & \multicolumn{2}{c|}{ Net plant efficiency } & \multicolumn{2}{c|}{$\begin{array}{c}\text { Capacity factor } \\
{[\%]}\end{array}$} \\
& 2015 & 2025 & Variation & 2015 & 2025 & 2015 & 2025 \\
\hline DNI 2000 & 526.6 & 551.3 & $+4.7 \%$ & $15.2 \%$ & $17.5 \%$ & $37.6 \%$ & $39.3 \%$ \\
DNI 2550 & 575.9 & 624.6 & $+8.4 \%$ & $14.9 \%$ & $16.6 \%$ & $41.1 \%$ & $44.6 \%$ \\
DNI 2900 & 639.7 & 683.6 & $+6.9 \%$ & $15.1 \%$ & $16.9 \%$ & $45.6 \%$ & $48.8 \%$ \\
\hline
\end{tabular}

TABLE III. - Electric production and system performance figures of a solar tower reference system for state of the art technology and estimations for 2025 [3].

\begin{tabular}{l|ccc|cc|cr|}
\hline & \multicolumn{2}{|c|}{$\begin{array}{c}\text { Net electrical output } \\
\text { [GWh / a] }\end{array}$} & \multicolumn{2}{c|}{ Net plant efficiency } & \multicolumn{2}{c|}{$\begin{array}{c}\text { Capacity factor } \\
\text { [\%] }\end{array}$} \\
& 2015 & 2025 & Variation & 2015 & 2025 & 2015 & 2025 \\
\hline DNI 2000 & 574.0 & 610.0 & $+6.2 \%$ & $14.4 \%$ & $17.1 \%$ & $43.7 \%$ & $46.4 \%$ \\
DNI 2550 & 598.4 & 644.0 & $+7.6 \%$ & $15.5 \%$ & $18.3 \%$ & $45.5 \%$ & $49.0 \%$ \\
DNI 2900 & 656.6 & 684.4 & $+4.2 \%$ & $15.6 \%$ & $18.2 \%$ & $50.0 \%$ & $52.1 \%$ \\
\hline
\end{tabular}

oil by molten salt in parabolic trough in 2025) by different sets of technical parameters. The capacity factor shown here is defined as the annual electricity energy provided by the power plant divided by the nominal capacity multiplied by 8760 hours. Tower systems are expected to have slightly higher efficiencies compared to parabolic trough systems and are capable to achieve higher capacity factors.

\section{5. - Cost}

Based on information of commercially built power plants on investments of the different components and subsystems as well as on typical surcharges for engineering, procurement, construction (EPC), and owner's cost for the project development, land preparation and other factors, investments cost for the defined trough and tower reference concepts were calculated as shown on the left in fig. 15 and fig. 16. Technology advancements and other factors that will be discussed later are expected to lead to a cost reduction until 2025 as presented on the right-hand side of the figures mentioned. The solar concentrator (that consists of a solar field in the case of the parabolic trough and of a heliostat field, a tower and a receiver for the tower system) has the major cost share 


\section{5}

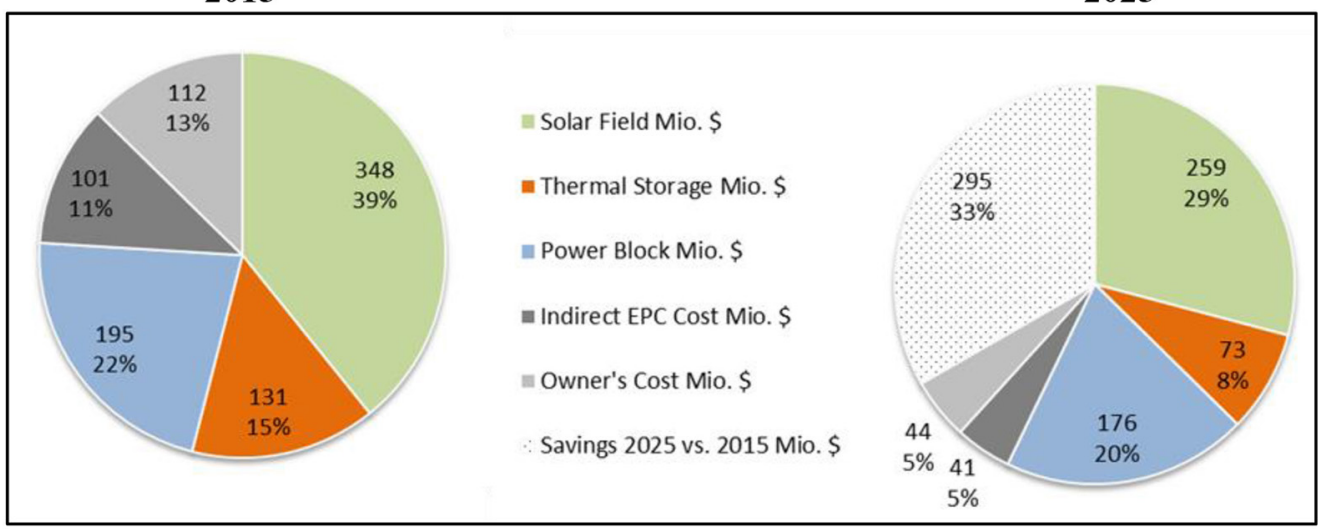

Fig. 15. - Comparison of CAPEX structure in 2015 and 2025 for $160 \mathrm{MW}$ parabolic trough power plant in Mio US\$ [3].

followed by the power block. Today, high surcharges in EPC and owners cost exist due the early market situation of the technology.

In order to compare the technologies, levelised cost of electricity (LCOE) are calculated using the following equation:

$$
\mathrm{LCOE}=\frac{C_{\text {invest }} \cdot\left(f_{\text {annuity }}+f_{\text {ins.,ann. }}\right)+C_{O \& M, a n n .}+C_{\text {elec }}+C_{f u e l}}{E_{\text {el,net,ann. }} \cdot f_{\text {avail }, \text { plant }}},
$$

with $C_{\text {invest }}$ being the capital investment, $C_{O \& M \text {,ann }}$ the annual operation and maintenance costs, $C_{\text {elec }}$ the annual electricity costs (if any), $C_{\text {fuel }}$ the annual fuel costs (if any),

2015

2025

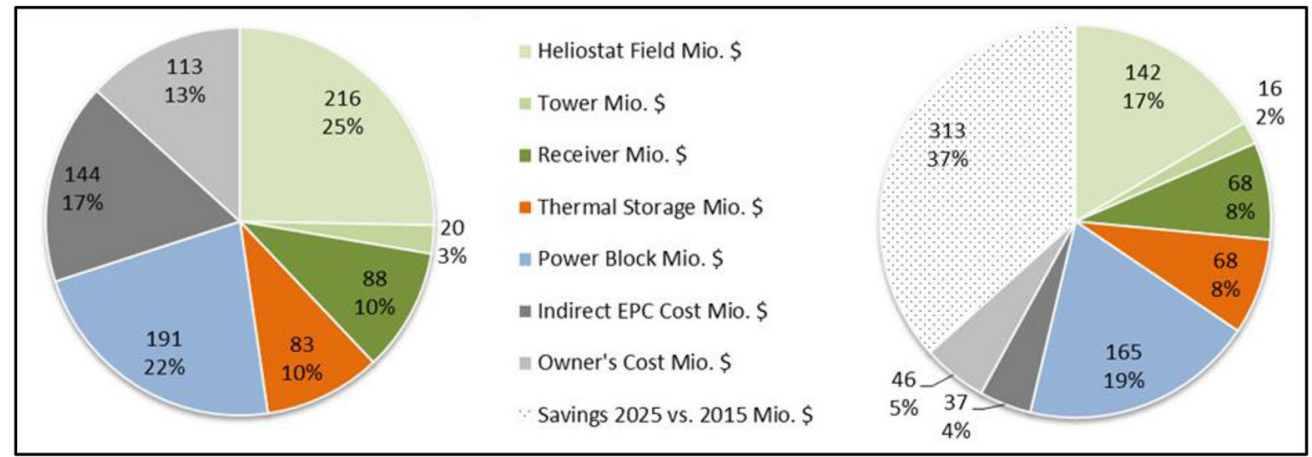

Fig. 16. - Comparison of CAPEX structure in 2015 and 2025 for $150 \mathrm{MW}$ solar tower power plant in Mio US\$ [3]. 


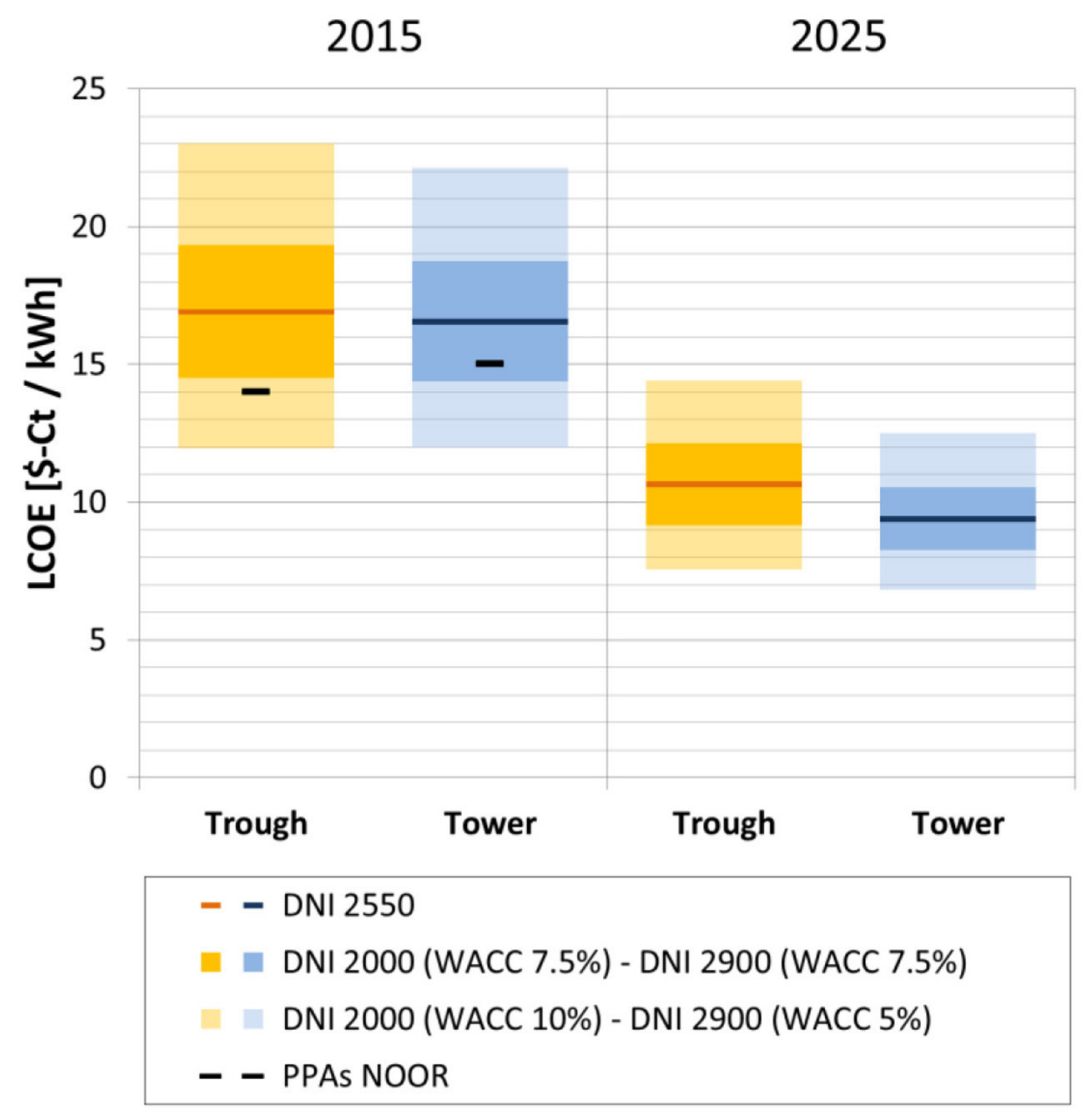

Fig. 17. - LCOE in 2015 and anticipated by experts for 2025 for parabolic trough and tower technology, different solar resource and financing conditions [3].

$E_{\text {el,net,ann }}$ the annual net electricity production, $f_{\text {annuity }}$ the weighted average cost of capital factor, $f_{\text {ins,ann }}$ the fraction of capital costs needed to cover the annual insurance costs and $f_{\text {avail,plant }}$ as the average annual plant availability.

The LCOE in US $\$$ cents $/ \mathrm{kWh}$ is compared in fig. 17 between trough and tower systems for the year 2015 and 2025. The colored margin shows the impact of a variation in the solar resource and in different financing conditions expressed by the factor weighted average cost of capital (WACC $=f_{\text {annuity }}+f_{\text {ins,ann }}$ ). In addition, electricity prices from power purchase agreements announced in 2015 of a trough and a tower plant to be built in Morocco at around $2500 \mathrm{kWh} / \mathrm{m}^{2}$ · a (Noor II \&III) are indicated in the picture. They are below the average expected value of the model calculation because financing costs of those projects where lower than assumed in the model calculations due to the availability of soft loans provided by development banks.

Cost of troughs and towers are very similar and are expected to be reduced significantly over the next decade. Trough technology may keep up with the tower system if 
it is possible to replace the thermal oil heat transfer fluid by molten salt as it is state of the art in tower technology already today. In 2015 CSP electricity prices range today between 12 and $20 \$ c e n t s / \mathrm{kWh}$ depending on financing and site conditions. In 2017 new CSP tower plants were offered in at rates of $7.3 \$$ cents $/ \mathrm{kWh}$ for a project in UAE with a DNI in the range of $2200 \mathrm{kWh} / \mathrm{m}^{2} \mathrm{aA}$ mojor reason for this are the low financing cost that could be achieved in this project. In Chile under the world's best solar resource of more than $3000 \mathrm{kWh} / \mathrm{m}^{2}$ a a price of less than $7 \$$ cents $/ \mathrm{kWh}$ was offered in the same year. These figures are surprisingly low and were anticipated by most experts only to be achievable in 2025. With this, CSP is approaching a level where first unsubsidized niche markets come into reach. This would help to accelerate the market growth of this technology and enable further cost reduction through learning.

5`1. Cost drivers. - As shown in the previous section, both parabolic trough and tower technology offer significant cost reduction potentials. However, the cost drivers for both technologies are quite different.

In general, LCOE reductions can be achieved either by improving the efficiency of the plant resulting in a higher electricity output or by reducing the costs (CAPEX and/or OPEX). Performance improvements as well as cost reductions contribute to the expected LCOE reductions considered by experts to be reachable until 2025. Figure 18 gives an overview of the main cost drivers for both technologies.

For trough technology, two major cost drivers can be identified. The increase of the temperature level enabled by the switchover to molten salt as HTF leads to a higher cycle efficiency of the power block and lower investment costs for the thermal storage due to the size reduction by more than $50 \%$ and the omission of a heat exchanger. Both effects together account for $1.9 \$$ cents $/ \mathrm{kWh}$ of LCOE reduction. The second important cost driver is the reduction of the solar field costs which is closely related to the usage of a trough collector with wider aperture and thus less collector units. Together both factors lead to LCOE reductions by $1.75 \$$ cents $/ \mathrm{kWh}$. The residual LCOE reduction is mainly achieved by the reduction of indirect EPC and owner's cost which are assumed to reduce proportional to the direct EPC cost.

For tower technology, the major cost driver is the gain of experience. Since many of the EPC contractors and project developers built their first tower projects in 2015, risk margins are still high, the commissioning phase is extended and the operational costs are higher (at least in the first years of operation) compared to mature trough technology. The lack of experience in 2015 is represented by three factors that are altered compared to the values for troughs. The plant availability is assumed to be only 0.93 in 2015 instead of 0.99 in order to account for longer outages due to unscheduled additional maintenance and replacement of broken components. Extended commissioning phases with replacement of broken components could be seen for several tower projects built until now. These additional maintenance and commissioning efforts are also responsible for the assumption of increased O\&M costs (2\% of CAPEX in 2015 instead of $1 \%$ in 2025). Indirect EPC costs are expected to be 9\%-points higher in 2015 due to additional risk surcharges within the supply chain. The mentioned three parameters are only altered 

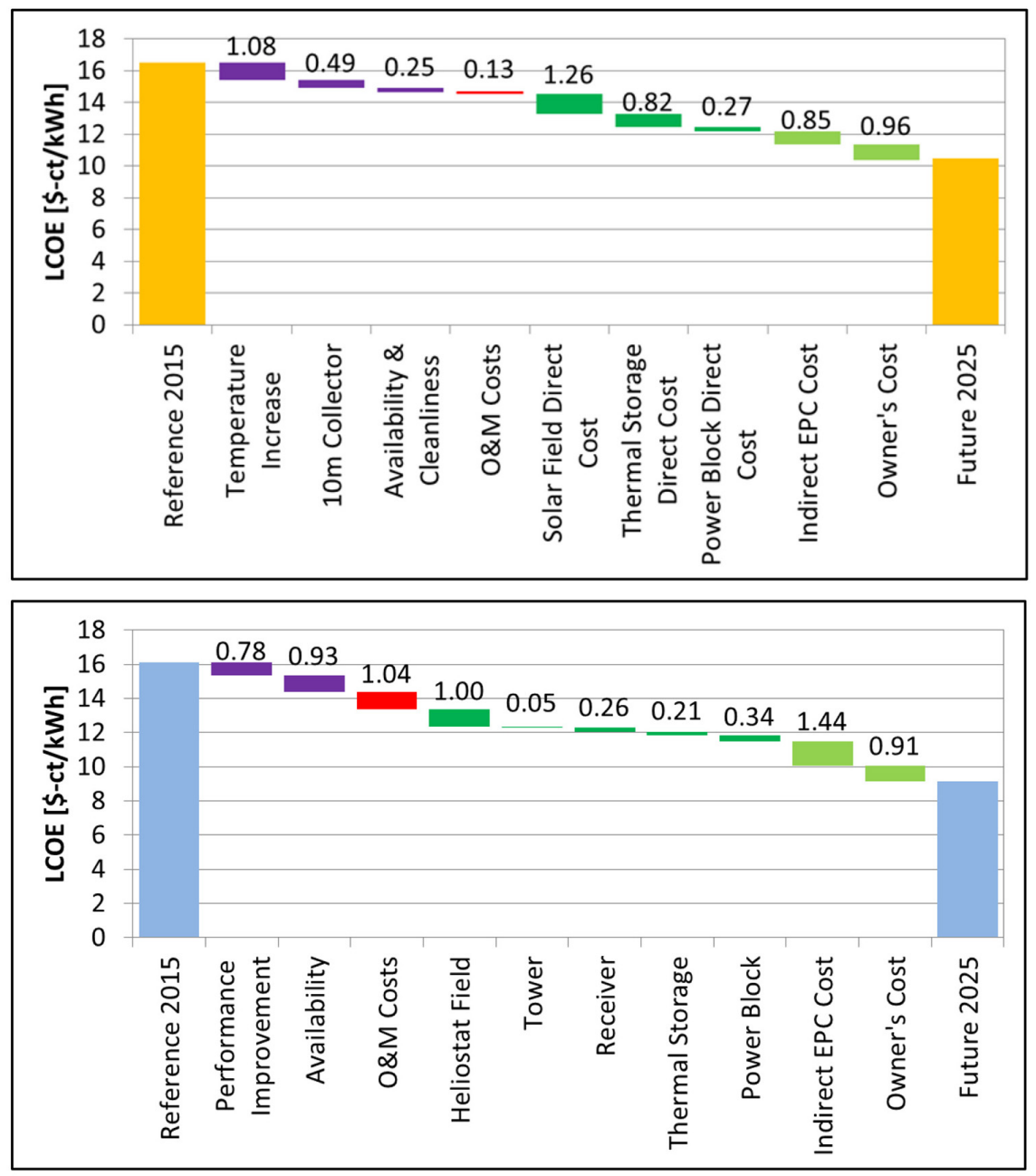

Fig. 18. - Contributing factors to the LCOE reduction from 2015 to 2025 for trough (top) and tower (bottom) systems: Performance improvement (violet), OPEX reduction (red), direct CAPEX reduction (dark green) and indirect CAPEX reduction (light green) [3].

for the year 2015. In 2025 the percentages for all indirect costs and availability are the same as for troughs which reduces the LCOE by $3.4 \$$ cents $/ \mathrm{kWh}$. With the exception of the heliostat field, the reduction of direct EPC costs is marginal. Performance improvements excluding availability have about half as much influence for tower technology $(0.78 \$$ cents $/ \mathrm{kWh})$ as for trough technology $(1.08+0.49=1.57 \$$ cents $/ \mathrm{kWh})$.

\section{6. - Summary and conclusion}

In solar thermal power systems, concentrating solar collectors provide high temperatures to a power engine to generate mechanical energy that is converted to electricity 
using a generator. It benefits from a mature power plant technology that has been optimized for almost a century using heat based on fossil or nuclear resources. Today, several options to concentrate direct sunlight are pursued. In linear concentrators like the parabolic trough or linear Fresnel systems, a line focus is achieved by one-axis tracking of $1 \mathrm{~d}$ curved reflector segments. In point focusing systems like towers systems, a point focus is generated by $2 \mathrm{~d}$ curved reflector segments and two-axis tracking. All options can be combined with thermal energy storage to provide energy on demand. Thus, technology concepts that are based on molten salt both as a storage and as a heat transfer fluid are in the focus of the current deployment. A significant cost reduction noticed in recent bids for commercial projects brings CSP power plants at the edge of full competitiveness. Further cost reductions appear to be feasible for both, trough and tower systems. Both technologies bear advantages and drawbacks that do not allow a clear preference for one technology today.

\section{REFERENCES}

The work has been compiled partly from previous publications of the author:

[1] Pitz-Paal Robert, "High Temperature Solar Concentrators", in Solar Energy Conversion and Photoenergy Systems, edited by Blanco Galvez Julian and Malato Rodriguez Sixto (Eolss Publishers, Oxford, UK) 2007.

[2] Pitz-Paal Robert, Giuliano Stefano and Wittmann Michael, "Concepts for Cost Reduction in CSP Power Plants", in Energie Erzeugung, Netze, Nutzung - Vorträge auf der DPG Frühjahrstagung, Berlin 16.-18.3.2015 (Deutsche Physikalische Gesellschaft, Berlin) 2015, pp. 42-45. ISBN 978-3-9811161-7-5.

[3] Dieckmann Simon, Dersch Jürgen, Lüpfert Eckhard, Pitz-Paal Robert, Giuliano Stefano and Puppe Michael, Assessment of the Cost Reduction Potential for Concentrating Solar Power Until 2025, study performed by the German Aerospace Center (DLR) for the International Renewable Energy Agency (IRENA) 2016. 